\title{
Risk Based Decision Algorithms for Management of COVID-19 Associated Rhino-orbital Mucormycosis
}

\author{
Neeti Kapre Gupta ${ }^{1} \cdot$ Madan Kapre $^{1} \cdot$ Harshkaran Gupta $^{1} \cdot$ Gauri Kapre Vaidya $^{1} \cdot$ Shripal Jani $^{2}$. \\ Sonal Meshram ${ }^{1} \cdot{\text { Sanjog } \text { Singh }^{2} \cdot \text { Vidula Kapre }^{3} \cdot \text { R. Ravi }^{4} \cdot \text { Vilas Tambe }^{3} \text { - Nirmala Thakkar }}^{1}$. \\ Anju Mundhada ${ }^{4}$
}

Received: 8 June 2021 / Accepted: 16 June 2021 / Published online: 30 July 2021

(C) Association of Otolaryngologists of India 2021

\begin{abstract}
Introduction COVID associated mucormycosis is a challenging problem with significant morbidity and mortality implications. COVID affliction, pre-existing medical conditions especially diabetes and steroid prescription are supposed contributors for development of this opportunistic fungal infection. Surgery remains the mainstay of treatment with adequate post-op anti-fungal therapy.

Materials and methods This is a retrospective analysis of prospectively maintained database of all surgical patients between March-May 2021 at a single centre. Prognosticators such as severity of COVID affliction, use of steroids, extent of rhino-orbital mucormycosis, extent of surgery and outcomes were studied. Descriptive statistics was used to
\end{abstract}

Supplementary Information The online version contains supplementary material available at https://doi.org/10.1007/ s12070-021-02692-9.

Neeti Kapre Gupta

neetikapregupta@gmail.com

1 Department of ENT and Head Neck Surgery, Neeti ENT and Head Neck Cancer Clinics, Neeti Gaurav Complex, Near Lokmat Square Ramdaspeth, Nagpur, Maharasthra 440010, India

2 Department of Oral and Maxillo-Facial Surgery, Neeti ENT and Head Neck Cancer Clinics, Neeti Gaurav Complex, Near Lokmat Square Ramdaspeth, Nagpur, Maharasthra 440010, India

3 Department of Anaesthesia and Critical Care Medicine, Neeti ENT and Head Neck Cancer Clinics, Neeti Gaurav Complex, Near Lokmat Square Ramdaspeth, Nagpur, Maharasthra 440010, India

4 Department of Pathology and Microbiology, Neeti ENT and Head Neck Cancer Clinics, Neeti Gaurav Complex, Near Lokmat Square Ramdaspeth, Nagpur, Maharasthra 440010, India analyse frequency of different variables and chi square test was used to analyse prognostic factors. $P$ value $<0.05$ were deemed significant.

Results 74 patients with an average 30 day follow-up were included. All patients had preceding COVID infection and $71.6 \%$ were diabetic and $25.7 \%$ were diagnosed during the course of their COVID treatment. Multiple sinus involvement being most common (52.61\%), 10.81\% underwent orbital exenteration and $24.32 \%$ palatectomies were performed. Revision surgeries were warranted in $16.21 \%$ patients. 9 patients succumbed to disease/ underlying medical and treatment related complications.

Conclusion We propose a risk assessment based on general condition of patient and severity of mucormycosis infection to decide appropriate strategy for surgical intervention. Early detection and timely and adequate surgery are essential pre-requisites to good outcomes.

Keywords Rhino-orbital · Mucormycosis ·

Risk assessment · COVID-19

\section{Introduction}

The COVID-19 pandemic, especially in its second wave has presented an unforeseen complication in the form of COVID associated mucormycosis. This life-threatening condition caused most often by Mucor Mucorales and is generally seen in post COVID patients with immune depleted status on account of either co-existing diabetes, renal disease and other comorbid medical conditions or due to injudicious use of steroids, not to mention Covid infection itself. It poses several challenges to the surgeon right from timing of surgical intervention, diagnosis and differentiation from other bacterial sinusitis, decision for 
extent of surgery and ideal approach, selection of systemic anti-fungals, monitoring and management of the general health and co-morbidities of these patients simultaneously. Needless to say therefore that treatment planning and execution requires a great deal of coordinated efforts between ENT, maxillo-facial and ophthalmic surgeons, infectious disease specialists, endocrinologists and intensive care experts. Central India, our place of practice is unequivocally the epicentre for this dual enemy of COVID19 disease and super-imposed rhino-orbital mucormycosis. There have been isolated case reports and small case series published on this topic so far, majority from the Indian subcontinent. [1-5] Therefore, through this article we wish to present results of our prospectively maintained database in attempt to understand disease presentation and recognise prognosticators for better treatment outcomes. Towards this aim, we have also proposed a scoring system, currently employed at our centre as a tool for risk assessment and decision making in rhino-orbital mucormycosis developing in the background of COVID-19.

\section{Etiopathogenesis}

The development of opportunistic fungal infection such as Mucormycosis with COVID 19 can probably be explained due to following reasons.

1. Thrombotic phenomenon causing direct vascular endothelial damage [6]

2. Decrease in $\mathrm{CD}+4$ and $\mathrm{CD}+8 \mathrm{~T}$ cells

3. Prolonged and/or injudicious steroid administration

4. Oxygen therapy (yet unproven)

Mucormycosis is a rare disease caused by Mucorales order fungi. Angio-invasion leading to micro-thrombosis and ischaemic necrosis are pathognomonic of this fungal infection. Before the COVID-19 pandemic, the prevalence of Mucor in India was 0.14/ 1000 population and paranasal sinuses and orbit being the most common sites involved. [7] There has been a dramatic rise in Mucormycosis cases in the country in the past few months. Over 2 crore Indians have been afflicted by COVID-19 disease. It is not unimaginable therefore that we are now facing an epidemic of Rhino-orbital Mucormycosis amidst the pandemic of COVID-19.

\section{Materials and Methods}

This is a retrospective analysis of a prospectively maintained database of surgical patients over the time period March-May 2021. All patients with confirmed diagnosis of mucormycosis on microbiological/ pathological examination and with a minimum 30 day follow-up, were included in the study. Demographic variables such as age, sex, co-morbidities were noted. Course of the COVID-19 disease was documented in detail including the symptomatology, results of COVID-19 RTPCR study, requirement of hospitalization/ oxygen/ ventilatory support, severity score on HRCT Chest and steroids prescribed. Blood biochemistry parameters such as Blood glucose levels, HbA1c, CRP, D-dimer, Kidney and liver function tests were also done as a part of pre-operative work-up. Clinical presentation, results of diagnostic investigations, treatment details and outcomes of Mucormycosis was noted in a dedicated proforma (Appendix 1 of supplementary material). Diagnosis was largely based on the clinical picture. Nasal endoscopy and micropathological studies were done to confirm diagnosis of fungal infection. However, negative fungal stain on the background of strong clinical suspicion was still considered for surgical exploration/ intervention. Extent of disease was assessed by imaging studies. Computed tomography of the paranasal sinuses was the most commonly ordered radiological investigation. Magnetic Resonance Imaging was requested for a suspected orbital or intracranial extension.

Pre-treatment evaluation was done in a joint clinic setting with intensivist, ENT surgeon, maxillofacial surgeon, pathologist and infectious disease specialist. A management plan was drawn up with special stress laid on timing of intervention and aggressiveness of approach (see Table 2 in results). Surgical approach most commonly used was combined endoscopic and external. Pure endoscopic approach was employed for early limited disease. In patients where suspicious necrotic tissue was seen at the sphenopalatine foramen and when the imaging revealed erosion/extension in the pterygopalatine fossa area, posterior wall of maxilla was punched out for ensuring complete disease clearance. Infra-structure maxillectomy was required for patients with frank palatal lesions. These patients then went in for dental prosthetic rehabilitation after full recovery.

Patients with frank orbital involvement were counselled in detail regarding possible need for orbital exenteration. For a non-seeing eye, open orbital exenteration using a Howarth-Lynch incision was performed. Lid sparing procedures were preferred unless there was frank involvement of the eyelid by the fungus. Eye globe preserving gross resection/ orbital debridement was performed in salvageable eyes.

All specimens were sent for histopathology and fungal culture. Regular out-patient cleaning was done in postoperative period.

Statistical Analysis: Descriptive statistics was used to analyse frequency of different variables and chi square test 
was used to analyse prognostic factors. $P$ value $<0.05$ were deemed significant.

\section{Results}

There were 74 patients presenting with rhino-orbital mucormycosis after COVID-19 disease during the months of March-May 2021. (Table 1) We have excluded 3 patients with disseminated disease (pulmonary/ gastro-intestinal) as they were not surgical candidates and 12 surgical patients since they had not completed the mandatory 30 day follow-up. The average age of the population was 51.76 years, youngest patient being 12 years of age and oldest 78 years old. The male to female ratio was 1.84:1. $71.6 \%$ of patients were known diabetics and further $25.7 \%$ were detected during COVID-19 treatment. There was 1 patient with haemato-lymphoid malignancy while 5 patients were suffering from renal failure. All patients were diagnosed with COVID-19 infection, preceding to the mucormycosis presentation. Ninety five percent were RTPCR positive and remaining 5\% were diagnosed on the basis of HRCT Chest findings with CORADS score 4 or $5.90 .5 \%$ patients were prescribed steroids for COVID-19 pneumonia. The most commonly prescribed steroid was methylprednisolone for an average duration of 7 days. The average timing of presentation of mucormycosis from COVID disease was 17.6 days. The most common clinical symptom was headache and sinus area pain (45.94\%), followed by dental pain $(27.03 \%)$, visual symptoms (16.22\%) and facial swelling/ paraesthesia (10.81\%) (Fig. 1). While evaluating extent of disease, multi-sinus involvement was seen most frequently (52.61\%). 20.27\% had intra-orbital and $4.1 \%$ intra-cranial extension. Bilateral disease was seen in $13.5 \%$. (Fig. 2).

Twelve patients were managed with purely endoscopic surgery on account of limited single sinus involvement. Remaining patients underwent combined external and endoscopic approach for complete clearance of disease. Orbital exenteration was done in 8 patients. Palatectomy

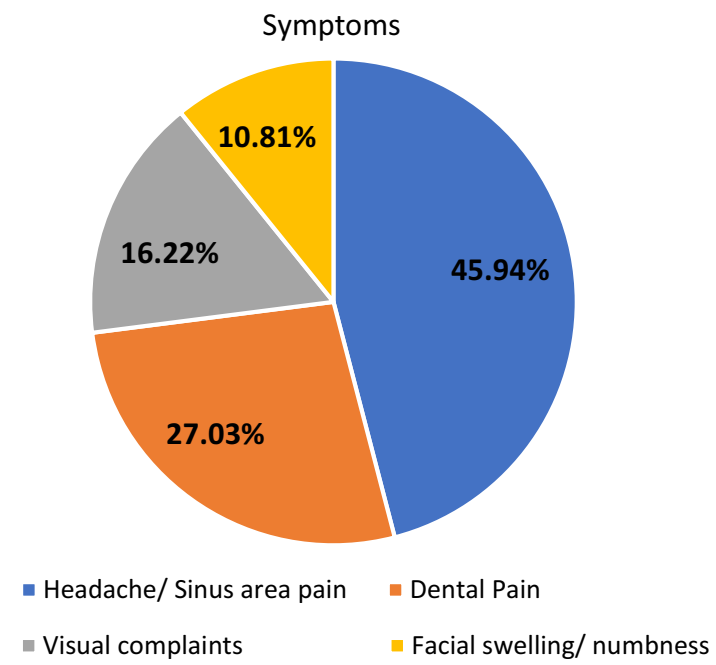

Fig. 1 Symptomatology of mucormycosis patients

was performed in 18 patients. $16.21 \%$ required revision surgeries for disease debridement (of these 3 patients were previously operated by the same group of surgeons \& remaining 9 were patients operated elsewhere initially and referred to us for revision surgery). Thus, revision surgery rate for patients managed throughout by our team was $4.62 \%$.

Mucor was the most commonly isolated fungus. (Fig. 3) 3 patients had aspergillus seen on microbiology, while 1 had candida. All patients were evaluated jointly by the surgeon, intensivist and infectious disease specialist to plan post-operative systemic anti-fungal therapy. Liposomal Amphotericin B was the drug of choice. Posaconazole was recommended in patients as second line anti-fungal. The average follow-up of our study was 5.2 weeks. 5 patients succumbed to their underlying medical condition/treatment related complications while 4 died due to disease progression. On univariate analysis extensiveness of mucormycosis $(p=0.009)$, need for orbital exentration $(p=0.003) \&$ renal failure $(p=0.011)$ were statistically significant prognosticators for mortality, where as diabetes $(p=0.209) \&$ palatal resection $(p=0.263) \&$ revision

Table 1 Distribution of patients according to study variables

\begin{tabular}{llll}
\hline Variable & Present (Number, Percentage) & Absent (Number, Percentage) & Total (Number, Percentage) \\
\hline COVID affliction & $74(100 \%)$ & $0(0 \%)$ & $74(100 \%)$ \\
Requirement of hospitalization/ Oxygen support & $51(68.9 \%)$ & $23(31.1 \%)$ & $74(100 \%)$ \\
Steroid prescribed for COVID & $67(90.5 \%)$ & $7(9.5 \%)$ & $74(100 \%)$ \\
Diabetes & $72(97.3 \%)$ & $2(2.7 \%)$ & $74(100 \%)$ \\
Renal Failure & $5(6.8 \%)$ & $69(93.2 \%)$ & $74(100 \%)$ \\
Haematolymphoid malignancy & $1(1.4 \%)$ & $73(98.6 \%)$ & $74(100 \%)$ \\
\hline
\end{tabular}


Fig. 2 Extent of involvement by rhino-orbital mucormycosis
Extent of involvement by Rhino-orbital Mucormycosis

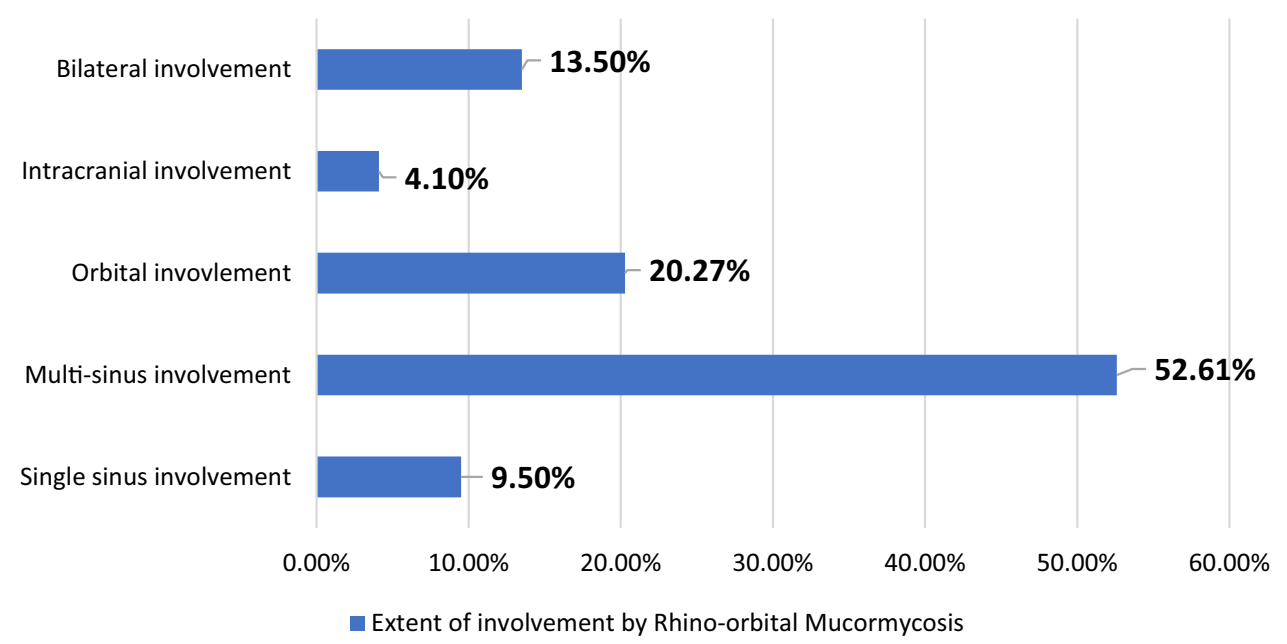

surgery requirement $(p=0.633)$ were not significant. However, purely endoscopic approach at initial surgery was seen to correlate with need for revision in a statistically significant manner $(p=0.001)$.

Based on clinical data of the above series, we propose a risk assessment scoring system to prognosticate severity of mucormycosis infection, and risk posed by deterioration of general medical condition on account of COVID-19 and other co-morbid states. This helps us to formulate strategies for timing and aggressiveness of interventional treatment of rhino-orbital mucormycosis (Tables 2, 3 and 4).

\section{Discussion}

The second wave of COVID-19 infection has posed a challenging problem for ENT surgeons across the country. Rhino-orbital mucormycosis raised its ugly head probably as a combined complication of COVID-19 disease itself, steroids administered as a part of treatment for COVID and pre-existing medical conditions such as diabetes, renal failure, haemato-lymphoid malignancies, etc. The association of mucormycosis with uncontrolled diabetes is proven and it is known to be an infection peculiar to diabetics, rarely seen in the normal population [8, 9]. India stands second with 77 million people with diabetes and another 36.5 million with prediabetes [10]. Before the COVID pandemic, prevalence of mucormycosis was $0.14 / 1000$ population, which is 80 times more than that seen in developed countries. Literature regarding safety of surgical intervention in a patient recovering from COVID-19 suggested that 6 weeks was a desired interim period. However, this life threatening disease required emergency surgery of the nose and paranasal sinuses. Prognosis in mucor infections is poor and time sensitive ranging between 33.3 and
$80 \%[11,12]$. A delay of even 6 days in initiating treatment doubles the 30-day mortality from $35 \%$ to $66 \%$ [13]. This implied significant risk to surgical team (contracting COVID infection due to prolonged closed space contact with patient's nasopharynx and airway) and to the patients themselves (risk of general anaesthesia amidst/immediately after cytokine reaction and risk of thrombo-embolic events during or after surgery, risk of worsening of renal functions/ previous medical conditions during anti-fungal treatment). Therefore, our centre proposed a risk assessment protocol aimed at guiding timing and aggressiveness in surgical treatments. We have put forth this series even in a nascent stage of our data with a wish to contribute to world literature. Further addition of numbers over the coming months and longer follow-up periods will strengthen the recommendations made. Guidelines for treatment of rhino-orbital mucormycosis is a need of the hour and we hope that systemic reviews of such series will play its part in improving the understanding of this new 'pandemic'.

In our series, there was a strong association between severe COVID affliction and development of fungal infection. The effect of COVID as discussed earlier is multi-pronged and attributable to depression of T-cell mediated immunity, thrombotic phenomenon promoting vascular endothelial damage, raised ferritin levels, disturbed glycaemic control during steroid therapy and injudicious use of antibiotics predisposing opportunistic fungal infections. In a recent review, 8 per cent of coronaviruspositive or recovered patients had secondary bacterial or fungal infections during hospital admission, with widespread use of broad-spectrum antibiotics and steroids [14]. $90.5 \%$ of our patients had received steroids for treatment of COVID pneumonia. Steroids were administered routinely for hospitalised patients. However, few patients with mild 

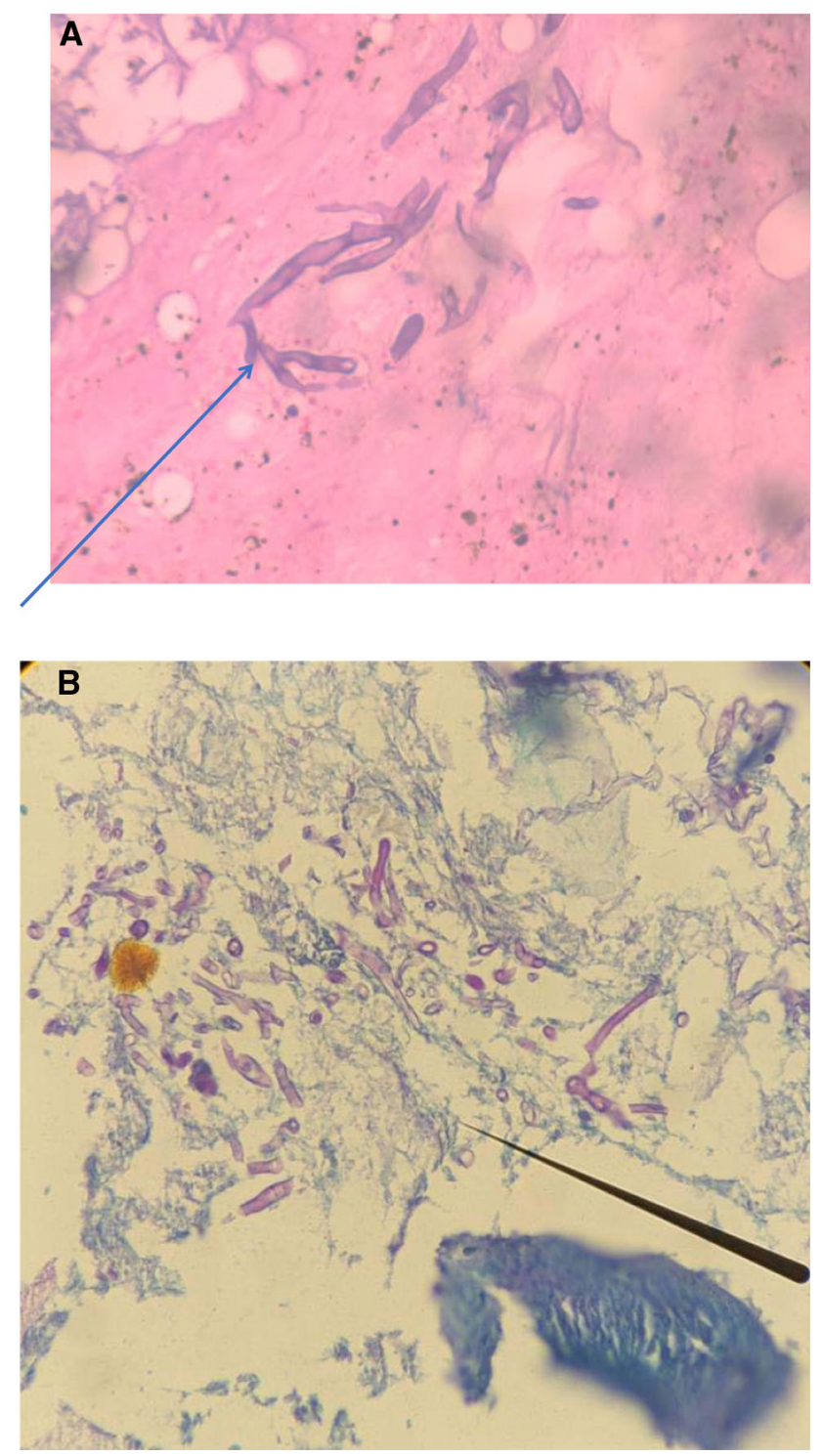

Fig. 3 a Photomicrograph of histopathological examination of tissue from maxillary sinus mucosa (Heamatoxylin and Eosin) showing broad aseptate hyphae in the background of ischaemic necrosis suggestive of Mucormycosis. b Periodic Acid Schiff stain from maxillary sinus showing Mucorales fungus

disease on home treatment also unfortunately received these medications. The National Institute of Health, according to the Randomised Evaluation of Covid-19 Therapy ('RECOVERY') Collaborative Group, recommends steroid use only in patients who are on a ventilator or require supplemental oxygen, but not in milder cases [15]. The guidelines specifically mention the risk of developing a secondary infection.

The average time of presentation of rhino-orbital mucormycosis after COVID infection was 17.6 days. In the initial period this interval was greater, in the range of 4-5 weeks. Headache, sinus area pain and nasal stuffiness were subtle symptoms with overlap with COVID infection and probably therefore were misdiagnosed. But as the awareness regarding this fungus increased amongst clinicians and lay public, the average time of presentation was much shorter (10-12 days). Headache, dental pain, orbital complaints, facial swelling/ numbness and nasal crusting were commonest symptoms in our series. Orbital complications, particularly loss of vision, were the most distressing symptom of the disease with maximum detriment to quality of life. In our analysis, we also noticed orbital involvement as a surrogate of extensive disease to adversely affect prognosis in a statistically significant manner. The etiopathogenesis of orbital involvement was more often central retinal artery occlusion (CRAO) than actual spread along the optic nerve [16-18]. Orbital exenteration was done only for patients with frank intra-conal involvement, complete occlusion of retinal artery rendering a non-salvageable non-seeing eye. There was 1 patient with bilateral eye involvement (complete blind right eye and only perception of light in left eye). We preserved the uninvolved eye globe while achieving gross $\mathrm{R} 0$ resection of involved peri-orbital tissues.

There has been much debate amongst ENT, Maxillofacial and general surgeons alike regarding most desirable surgical approach. Based on our experience, the authors recommend this decision to be case based. Factors that need to be taken into consideration were symptomatology, radiological extent of disease and the general condition of the patient for tolerating radical/ repeat surgical procedures. Intra-operative findings compelling the surgeons to change their approach was seen in $9.46 \%$ cases. This was on account of unanticipated disease that was under-estimated by radiology. Common areas for this were- anterolateral wall of maxilla with surreptitious spread of disease along the infra-orbital foramen, pterygo-palatine fossa and retro-maxillary soft tissue and far lateral end of the frontal sinus. We feel that these areas may not be easily approached by the occasional endoscopic surgeon and stand the risk of leaving behind residual disease.16.2\% required revision surgeries for disease debridement (only 3 cases inhouse and 9 surgeries for patients previously operated outside). We feel that our strategy for combined approach was instrumental in the low incidence of revision debridements (only 4.62\%).

Patients where palatal resections/ orbital exenterations were warranted, an opinion from team of reconstructive surgeons and prosthodontists was sought. On an average 1 month after completion of anti-fungal therapy was agreed upon as a suitable time to initiate rehabilitation, provided if the general condition of the patient allowed it.

Post-surgery, patients were evaluated by an infectious disease specialist to recommend appropriate anti-fungal regimen. The patients then completed this medical treatment under the care of a physician/ intensive care expert. In 
Table 2 Severity of COVID-19 affliction/ immune-compromise on account of underlying co-morbidities

\begin{tabular}{llll}
\hline Clinical Parameter & & & \\
\hline Value $=$ Score & Mild & Moderate & Severe \\
\hline COVID RTPCR Ct value & Negative $=0$ & $<24=1$ & $>24=2$ \\
CT Chest severity score & $0-8=0$ & $8-14=1$ & $>14=2$ \\
Hospitalization & Not required $=0$ & Required $=1$ & Required ICU $=2$ \\
Hypoxia & No $=0$ & Only O2 support $=1$ & Ventilation support $=2$ \\
Cytokine storm & None $=0$ & & Yes $=2$ \\
$\begin{array}{l}\text { Mean fasting blood } \\
\text { glucose }\end{array}$ & $<150=0$ & $150-300=1$ & $>300=2$ \\
HbA1c & $<7=0$ & $7-8=1$ & $>8=2$ \\
Radiation therapy & None $=0$ & $3-6$ months ago $=1$ & $<3$ months ago $=2$ \\
Haemato-lymphoid & Under remission, currently no & Pancytopenia, but & Pancytopenia with severe \\
$\quad$ malignancies & pancytopenia $=0$ & neutropenia $>1000 / \mathrm{dl}=1$ & neutropenia $<1000 /$ dl $=2$ \\
Steroid therapy & None $=0$ & $</=10$ days $=1$ & $>10$ days $=2$ \\
Chronic renal failure & None $=0$ & & Yes $=2$
\end{tabular}

$<6=$ Low risk, $6-11=$ Intermediate risk, $>12=$ High risk

Table 3 Risk assessment score based on extent of mucormycosis

\begin{tabular}{lcccc}
\hline $\begin{array}{c}\text { Extent of } \\
\text { Mucormycosis }\end{array}$ & $\begin{array}{c}\text { Nasal cavity/ single sinus } \\
\text { involvement }=1\end{array}$ & $\begin{array}{c}\text { Multiple sinuses } \\
\text { involved }=2\end{array}$ & $\begin{array}{c}\text { Orbital } / \text { Palatal/ } \\
\text { Facial }=3\end{array}$ & $\begin{array}{c}\text { Bilateral disease/ } \\
\text { Intracranial }=4\end{array}$ \\
\hline
\end{tabular}

$<1=$ Low severity disease, $1-2=$ Intermediate severity disease, $>3=$ High severity disease

Table 4 summarizes strategy for timing and aggressiveness of surgical intervention based on above findings

\begin{tabular}{|c|c|c|}
\hline $\begin{array}{l}\text { Patient Risk } \\
\text { score }\end{array}$ & $\begin{array}{l}\text { Disease Severity } \\
\text { score }\end{array}$ & Policy on intervention \\
\hline High & High & $\begin{array}{l}\text { As soon as intensivist stabilises medical condition, aggressive approach with intensive post-op monitoring as } \\
\text { mortality associated with disease progression/ recurrence and treatment related/ medical comorbidity } \\
\text { complications is high }\end{array}$ \\
\hline High & Low & $\begin{array}{l}\text { Await minimum } 2 \text { weeks from COVID disease to ensure recovery from cytokine storm and related medical } \\
\text { complications, aggressive approach and intensive post-op monitoring for medical complications }\end{array}$ \\
\hline Low & High & Immediate intervention and aggressive approach with suitable anti-fungal treatment regime \\
\hline Intermediate & Intermediate & $\begin{array}{l}\text { Intervene as soon as intensivist clears safety form COVID disease, aggressive surgical clearance and } \\
\text { suitable anti-fungal regime with close monitoring for disease and treatment related complications }\end{array}$ \\
\hline Low & Low & $\begin{array}{l}\text { Await } 2 \text { weeks from COVID disease to ensure adequate medical recovery, adequate surgical clearance and } \\
\text { suitable anti-fungal regime }\end{array}$ \\
\hline
\end{tabular}

a randomized controlled trial of 201 patients with invasive mold disease, liposomal amphotericin used at $3 \mathrm{mg} / \mathrm{kg} / \mathrm{day}$ was equally effective but safer and better tolerated than 10 $\mathrm{mg} / \mathrm{kg} /$ day dose amphotericin [19]. We too recommended Liposomal Amphotericin B to our patients. However, during these months, there was a severe shortage of Amphotericin in the country. The patients were therefore triaged based on extent/ severity of disease. Patients with intracranial / orbital involvement, extensive bilateral disease/ retro-maxillary extension and recurrent disease were given preference. Patients with limited disease were sometimes shifted to oral antifungal therapy after 5-7 days.

During the SARS-CoV infection spread in 2003, the incidence of fungal infection was 14.8-27 per cent, and it was the main cause of death for severe acute respiratory syndrome patients, accounting for 25-73.7 \% in all causes of death [20-22]. Thus, we need to maintain continued caution and longer follow-up periods can provide more 
convincing data regarding outcomes. Nevertheless, complete resection of invaded and necrosed tissues is an essential pre-requisite. There is ample literature to support that aggressive surgical approach has been shown to improve survival $[23,24]$.

Therefore, we recommend early detection, high index of clinical suspicion, timely intervention, achieving maximum clearance while minimizing morbidity as the most appropriate surgical strategy.

\section{Conclusion}

COVID associated rhino-orbital mucormycosis is a challenging disease with significant mortality and morbidity. Although, more often seen in patients with pre-existing comorbidities and severe disease, we also have identified disease in young, otherwise healthy individuals, which remains a cause of concern. Early detection, high index of clinical suspicion, timely intervention, achieving maximum clearance while minimizing morbidity as the most appropriate surgical strategy.

Authors' Contributions Not applicable.

Funding Not applicable.

Data Availability Not applicable.

\section{Declarations}

Conflict of Interest The author declare that they have no conflict of interest.

\section{Ethics Approval NA}

Consent to Participate Not applicable.

Consent for Publication Not applicable.

\section{References}

1. Mehta S, Pandey A (2020) Rhino-orbital mucormycosis associated with COVID-19. Cureus 12(9):e10726. https://doi.org/10.7759/cureus.10726

2. Sarkar S, Gokhale T, Choudhury SS, Deb AK (2021) COVID-19 and orbital mucormycosis. Indian J Ophthalmol 69:1002-1004

3. Garg D, Muthu V, Sehgal IS, Ramachandran R, Kaur H, Bhalla A, Puri GD, Chakrabarti A, Agarwal R (2021) Coronavirus disease (Covid-19) associated mucormycosis (CAM): case report and systematic review of literature. Mycopathologia 86(2):289-298. https://doi.org/10.1007/s11046-021-00528-2 (Epub 2021 Feb 5. PMID: 33544266; PMCID: PMC7862973)

4. Revannavar SM, Supriya PS, Samaga L et al (2021) COVID-19 triggering mucormycosis in a susceptible patient: a new phenomenon in the developing world? BMJ Case Rep 14:e241663. https://doi.org/10.1136/bcr-2021-241663
5. Saldanha M, Reddy R, Vincent MJ (2021) Paranasal mucormycosis in COVID-19 patient. Indian J Otolaryngol Head Neck Surg. 22:1-4. https://doi.org/10.1007/s12070-021-02574-0 (Epub ahead of print. PMID: 33903850; PMCID: PMC8060684)

6. Sweeney JM, Barouqa M, Krause GJ, Gonzalez-Lugo JD, Rahman S, Gil MR (2020) Evidence for secondary thrombotic microangiopathy in COVID-19; medRxiv preprint https://doi.org/10.1101/2020.10.20.20215608

7. Chakrabarti A, Dhaliwal M (2013) Epidemiology of mucormycosis in India. Curr Fungal Infect Rep 7:287-292. https://doi.org/10.1007/s12281-013-0152-z

8. Unnikrishnan R, Misra A (2020) Infections and diabetes: risks and mitigation with reference to India. Diabetes Metab Syndr 14(6):1889-1894. https://doi.org/10.1016/j.dsx.2020.09.022

9. Pereira N, Andrade P, Laura SA, Abreu C, Figueiredo R, Silva R (2013) Rhino-orbito-cerebral mucormycosis in a diabetic patient with Idiopathic CD4+ Lymphocytopenia. Adv Infect Dis 3:248-252. https://doi.org/10.4236/aid.2013.34037

10. International Diabetes Federation (2015) IDF diabetes atlas, 2nd edn. International Diabetes Federation, Brussels, Belgium

11. Scheckenbach K, Cornely O, Hoffmann TK, Engers R, Bier H, Chaker A, Greve J, Schipper J, Wagenmann M (2010) Emerging therapeutic options in fulminant invasive rhinocerebral mucormycosis. Auris Nasus Larynx 37(3):322-328. https://doi. org/10.1016/j.anl.2009.09.001 (Epub 2009 Oct 25 PMID: 19857939)

12. Jung SH, Kim SW, Park CS, Song CE, Cho JH, Lee JH, Kim NS, Kang JM (2009) Rhinocerebral Mucormycosis: consideration of prognostic factors and treatment modality. Auris Nasus Larynx 36(3):274-279. https://doi.org/10.1016/j.anl.2008.07.003 (Epub 2008 Sep 10 PMID: 18786790)

13. Sen M, Lahane S, Lahane TP, Parekh R, Honavar SG (2021) Mucor in a viral land: a tale of two pathogens. Indian J Ophthalmol 69(2):244-252. https://doi.org/10.4103/ijo.IJO_3774_20 (PMID:33463566; PMCID:PMC7933891)

14. Rawson TM, Moore LSP, Zhu N, Ranganathan N, Skolimowska K, Gilchrist M, Satta G, Cooke G, Holmes A (2020) Bacterial and fungal coinfection in individuals with coronavirus: a rapid review to support COVID-19 antimicrobial prescribing. Clin Infect Dis 71(9):2459-2468. https://doi.org/10.1093/cid/ciaa530 (PMID:32 358954; PMCID:PMC7197596)

15. RECOVERY Collaborative Group, Horby P, Lim WS, Emberson JR, Mafham M, Bell JL, Linsell L, Staplin N, Brightling C, Ustianowski A, Elmahi E, Prudon B, Green C, Felton T, Chadwick D, Rege K, Fegan C, Chappell LC, Faust SN, Jaki T, Jeffery K, Montgomery A, Rowan K, Juszczak E, Baillie JK, Haynes R, Landray MJ (2021) Dexamethasone in hospitalized patients with Covid-19. N Engl J Med 384(8):693-704. https://doi.org/10.1056/NEJMoa2021436 (Epub 2020 Jul 17. PMID: 32678530; PMCID: PMC7383595)

16. Montesel A, Bucolo C, Mouvet V, Moret E, Eandi CM (2020) Case report: central retinal artery occlusion in a COVID-19 patient. Front Pharmacol 23(11):588384. https://doi.org/ 10.3389/fphar.2020.588384 (PMID:33424598;PMCID:PMC7785811)

17. Acharya S, Diamond M, Anwar S, Glaser A, Tyagi P (2020) Unique case of central retinal artery occlusion secondary to COVID-19 disease; IDCases. 21: e00867. Published online 2020 Jun https://doi.org/10.1016/j.idcr.2020.e00867 PMCID: PMC 7301806

18. Dumitrascu OM, Volod O, Bose S, Wang Y, Biousse V, Lyden PD (2020) Acute ophthalmic artery occlusion in a COVID-19 patient on apixaban. J Stroke Cerebrovasc Dis 29(8):104982. https://doi.org/10.1016/j.jstrokecerebrovasdis.2020.104982 (Epub 2020 May 23. PMID: 32689586; PMCID: PMC7 245224) 
19. Muthu V, Agarwal R, Dhooria S, Sehgal IS, Prasad KT, Aggarwal AN et al (2021) Has the mortality from pulmonary mucormycosis changed over time? a systematic review and metaanalysis. Clin Microbiol and Infect. https://doi.org/ 10.1016/j.cmi.2020.12.035

20. Zhang Y, Li WX, Huang KW, Cao ZX, Hao JY (2003) Hospital acquired pneumonia occurring after acute stage of the serious SARS and its treating strategies. Chin $\mathrm{J}$ Nosocomiol 11:1081-1087

21. Yin CH, Wang C, Tang Z, Zhang SW, Wang BS (2004) Clinical analysis of 146 patients with critical severe acute respiratory syndrome in Beijing areas. Clin J Emerg Med 1:12-14

22. Li CS, Pan SF (2003) Analysis and causation discussion of 185 severe acute respiratory syndrome dead cases [in Chinese]. Zhongguo Wei Zhong Bing Ji Jiu Yi Xue 15:582-584
23. Elinav H, Zimhony O, Cohen MJ, Marcovich AL, Benenson S (2009) Rhinocerebralmucormycosis in patients without predisposing medical conditions: a review of the literature. Clin Microbiol Infect 15:693-697

24. Goldstein EJ, Spellberg B, Walsh TJ, Kontoyiannis DP, Edwards J Jr, Ibrahim AS (2009) Recent advances in the management of mucormycosis: from bench to bedside. Clin Infect Dis 48:1743-1751

Publisher's Note Springer Nature remains neutral with regard to jurisdictional claims in published maps and institutional affiliations. 